\title{
Tipos psicológicos y estilos de aprendizaje de los estudiantes que ingresan a Medicina en la Pontificia Universidad Católica de Chile
}

\author{
Marcela Bitran $C^{1}$, Denisse Zúñiga $P^{2}$, Montserrat Lafuente $G$, \\ Paola Viviani $\mathbf{G}^{3}$, Beltrán Mena $\mathbf{C}$. \\ Psychological types and learning \\ styles of students entering \\ medical school at the Pontificia \\ Universidad Católica de Chile
}

Background: Psychological type and learning style influence the way students perceive and process information. However, research in medical education in Chile still does not put enough emphasis in the study of these variables. Aim: To characterize the psychological types and learning styles of the students admitted to a Medical School. Subjects and Methods: The Myers Briggs Type Indicator (MBTI) and the Kolb's Learning Styles Inventory (IEA) were administered to the 270 students admitted from 2000 to 2002 to the medical school of the Pontificia Universidad Católica de Chile. Results: Fifty five percent of our students are concentrated in 4 of the 16 psychological types described. These students are characterized by the ability to base their decisions upon logical and objective reasoning (Thinking [TD and to face life in a structured and decided way (Judging (JD). Only $10 \%$ of the students have preferences opposite to $\mathrm{T}$ and $\mathrm{J}$. These students base their decisions on the preservation of harmony and teamwork (Feeling [F]) and have a flexible attitude towards life (Perceiving $[\mathrm{PI}$. The remaining 35\% have types with pairs of preferences TP and FJ. With regard to learning styles, more than two thirds of our students are Assimilators or Convergers. These learners tend to assimilate large amounts of information and abstract the main concepts, rather than to pay attention to concrete details. In general, our students are more reflective than active; they evaluate thoroughly all alternatives before making a decision. Conclusions: The psychological types and learning styles of medical students cluster around specific patterns whose features may either favor or hamper a specific learning. Knowledge of the differences in psychological types and learning styles of students may provide teachers with a new and valuable tool for improving learning and contributing to the academic success of students (Rev Méd Chile 2003; 131: 1067-78).

(Key Words: Edutacion, medical, undergraduate; Learning; Psychological test; Schools, medical)

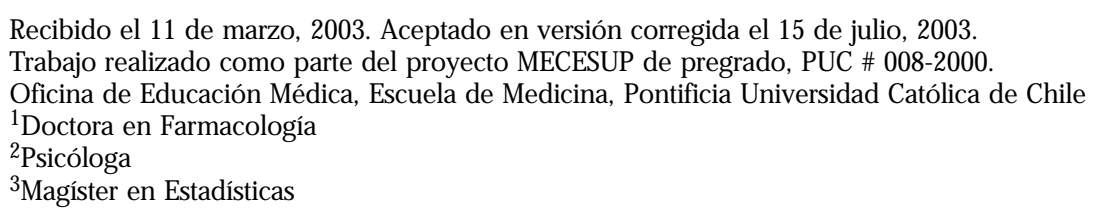

Correspondencia a: Marcela Bitran C. Oficina de Educación Médica, Escuela de Medicina, Pontificia Universidad Católica de Chile. Quito 41, Depto. D, Santiago Centro. Fono: 354-3811; Fax: 633-1457. E mail: mbitran@med.puc.cl 
$\mathrm{N}_{\mathrm{in}}$ uestra personalidad y estilo de aprendizaje influyen en la manera habitual en que percibimos y procesamos la información durante el aprendizaje ${ }^{1}$. Estas variables extraacadémicas son objeto de estudio en escuelas de medicina de Europa y Estados Unidos de Norteamérica por su importancia en el desempeño académico, la elección de especialidad y la satisfacción y eficiencia de sus alumnos ${ }^{1-7}$. En Chile, en cambio, hay muy pocos estudios publicados al respecto. La investigación en Educación Médica en nuestro país se ha centrado más bien en los antecedentes académicos de admisión (ej: resultado en la Prueba de Aptitud Académica (PAA), pruebas de Conocimientos Específicos y Notas de Enseñanza Media) y su correlación con el desempeño de los estudiantes durante la carrera-12. Este abordaje es insuficiente para las escuelas de medicina, ya que los estudiantes que ingresan a ellas suelen tener un rendimiento muy homogéneo en las pruebas de admisión ${ }^{8,13}$.

Nos interesó, por tanto, conocer las características de personalidad y estilos de aprendizaje de nuestros estudiantes y determinar cómo éstos se relacionan con su desempeño académico, aproximación al aprendizaje y proyección profesional. Para estudiar las características de personalidad escogimos el indicador de Tipos Psicológicos de Myers Briggs (MBTI), por ser un instrumento científicamente validado 14,15 , que permite detectar diferencias psicológicas entre individuos clínicamente normales ${ }^{15}$. Para mayor claridad, en adelante nos referiremos a las "caracteństicas de personalidad" con el término técnico tipos psicológicos.

Para conocer los estilos de aprendizaje usamos el Inventario de Estilos de Aprendizaje de Kolb (IEA), instrumento que detecta diferencias en el estilo de procesamiento de la información y que ha sido ampliamente utilizado y validado en medios académicos $21,24,30$.

Diseñamos un estudio longitudinal de 9 años de duración, llamado Psimed 21, para evaluar si los tipos psicológicos y los estilos de aprendizaje son variables relevantes para el desempeño académico de los estudiantes de medicina. En particular, nos interesó determinar si los tipos y estilos de los alumnos se asocian de manera consistente con su desempeño en determinadas asignaturas o actividades que requieren de conocimientos, habilidades o actitudes específicos. Nuestra hipótesis de trabajo es que los estudiantes tendrán mejor rendimiento en aquellas actividades cuyo objetivo y metodología apelen a las fortalezas distintivas de sus tipos psicológicos y estilos de aprendizaje. Estos individuos, sobresalientes en ciertas asignaturas o actividades, tendrán, por el contrario, mayores dificultades al enfrentarse a desafíos académicos que involucren el uso de otras habilidades 0 actitudes que no les son tan propias.

El estudio Psimed 21 se inició el año 2000 y contempla el seguimiento individual de todos los estudiantes de las cohortes 2000, 2001 y 2002. En el dossier de cada estudiante se registra: 1) su perfil de ingreso (características biodemográficas y académicas; tipo psicológico y estilo de aprendizaje), 2) su desarrollo a lo largo de la carrera (estabilidad de su tipo psicológico y estilo de aprendizaje, evolución de su rendimiento académico y ocurrencia de situaciones académicas o personales especiales) y 3) su situación profesional a dos años de su egreso. Todos los alumnos reciben los reportes individuales de sus resultados en los tests MBTI e IEA, junto con la información y explicaciones necesarias para su comprensión. La entrega de los reportes no está concebida como una intervención experimental, sino que responde a una decisión de carácter ético. En todo caso, dadas las características del MBTI y el Kolb, estimamos improbable que la simple lectura de los informes modifique las conductas de los estudiantes.

$\mathrm{Si}$ este estudio permite establecer, de manera fundada, que los tipos psicológicos y los estilos de aprendizaje son variables relevantes para el desempeño y desarrollo académicos de los estudiantes de medicina, contaremos con una base racional para optimizar la formación de nuestros alumnos. Las acciones a tomar dependerán de los hallazgos específicos del Psimed 21 y podrían incluir desde modificaciones metodológicas hasta intervenciones puntuales con grupos de estudiantes o docentes. El espíritu que debiera orientar estas acciones es el uso constructivo de las diferencias individuales. Esto pasa, entre otras cosas, por revisar la idoneidad de las metodologías de enseñanza utilizadas y determinar si éstas son adecuadas y suficientemente variadas para llegar a todos nuestros estudiantes. Además, junto con requerir de nuestros alumnos el dominio de múltiples saberes, habilidades y actitudes, debe- 
mos estar en condiciones de ofrecerles instancias para fortalecer aquellas características personales menos desarrolladas que también son necesarias para su éxito profesional y personal ${ }^{16}$.

Los resultados presentados en este trabajo constituyen la fase inicial del estudio Psimed 21 y corresponden a lo que hemos llamado el perfil de ingreso de nuestra población. Este perfil incluye características biodemográficas, académicas, tipos psicológicos y estilos de aprendizaje de los estudiantes de las cohortes 2000, 2001 y 2002, al ingreso a la carrera de medicina.

\section{Material y MÉTodo}

Población. Todos los estudiantes de las cohortes de ingreso 2000, 2001 y $2002(n=270)$ de la Escuela de Medicina de la Pontificia Universidad Católica de Chile (PUC). El perfil de estos estudiantes (Tabla 1) es similar al de los estudiantes que ingresaron hace una década ${ }^{17}$, excepto porque hoy entran proporcionalmente más mujeres y más estudiantes de colegios particulares (Tabla 1). Comparados con los estudiantes que ingresan a las distintas carreras de la PUC, los de medicina tienen un Puntaje de Selección más alto $(769 \pm 10$ [medicina] vs $707 \pm 36$ puntos [PUC], p <0,01) y menor proporción de estudiantes de la Región Metropolitana [67\% [medicina] vs $80 \%$ [PUC], p $<0,002)^{18,19}$. Según su rendimiento promedio en la PAA nuestros estudiantes se ubican en 1\% superior de la distribución normal nacional $(\mathrm{z}=0,99)^{20}$.

Instrumentos. Las características de personalidad se determinaron con el Indicador de Tipos Psicológicos de Myers Briggs (MBTI, forma $G$, versión en Español) ${ }^{14}$ y los estilos de aprendizaje con el Inventario de Estilos de Aprendizaje de Kolb (IEA, versión en Español ${ }^{21}$. Los instrumentos se aplicaron durante la primera semana de clases, previo consentimiento informado de los estudiantes.

Indicador de Tipos Psicológicos de Myers Briggs (MBTI). El MBTI, basado en la Teoría de Tipos psicológicos de Carl Jung 22 , permite detectar diferencias en la conducta de personas psicológicamente normales de acuerdo a: 1) su fuente de motivación, 2) su modo de percibir la información, 3) su modo de utilizar la información para tomar decisiones y 4) su postura frente al mundo (Figura 1).

Tabla 1. C aracterísticas de los jóvenes que ingresan a medicina

\begin{tabular}{|lcc|}
\hline & $\begin{array}{c}\text { Cohortes 1990-1992 } \\
(\mathrm{n}=193)\end{array}$ & $\begin{array}{c}\text { Cohortes 2000-2002 } \\
(\mathrm{n}=270)\end{array}$ \\
\hline Antecedentes biodemográficos & & \\
$\quad$ Edad (años \pm SD) & $18,3 \pm 1,1$ & $18,8 \pm 1,0$ \\
Mujeres & $35 \%$ & $47 \% *$ \\
Primogénitos & $\mathrm{S} / \mathrm{A}$ & $52 \%$ \\
$\mathrm{n}$ - hermanos & $\mathrm{S} / \mathrm{A}$ & $2,2 \pm 1,3$ \\
Antecedentes académicos & & \\
Puntaje de selección (puntos $\pm \mathrm{SD})$ & $760 \pm 11$ & $769 \pm 10$ \\
Estudios universitarios previos & $18 \%$ & $14 \%$ \\
Primera postulación a medicina & $\mathrm{S} / \mathrm{A}$ & $69 \%$ \\
Colegio particular & $73 \%$ & $83 \% *$ \\
Colegio municipalizado & $12 \%$ & $13 \%$ \\
Región Metropolitana & $73 \%$ & $67 \%$ \\
\hline
\end{tabular}

${ }^{*} p<0,03$ respecto del valor correspondiente de los estudiantes de las cohortes 1990-1992.

S/A: sin antecedentes. 


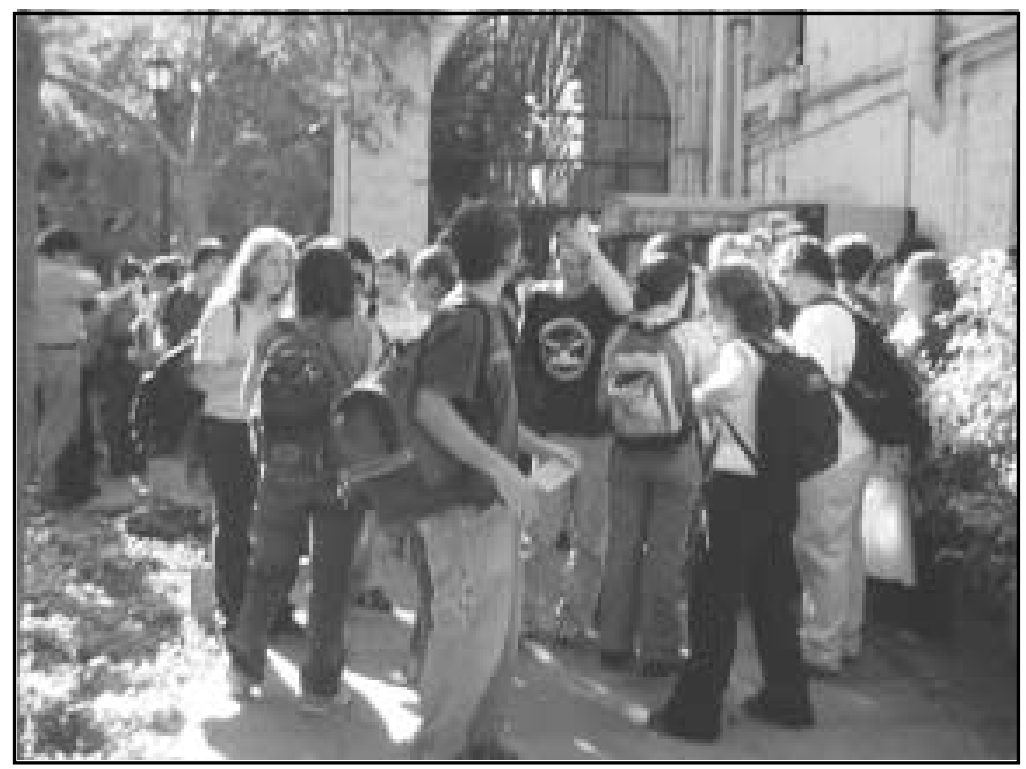

La personalidad y el estilo de aprendizaje de los estudiantes inciden en su desempeño académico y adaptación a la vida universitaria (alumnos de medicina en la primera semana de clases; fuente: Centro de Estudiantes de medicina, PUC).

El MBTI, a diferencia de otros instrumentos, no mide rasgos de personalidad sino preferencias por uno u otro polo de estas 4 dimensiones psicológicas $^{14}$. Los polos opuestos de la primera dimensión son Extraversion (E) e Introversion (I). Para un individuo con preferencia $\mathrm{E}$, la fuente de motivación está en el mundo extemo: en el contacto con las cosas y personas; si su preferencia es I, su motivación se origina principalmente de su mundo interior. En cuanto a la percepción de la información, las personas con preferencia Sensing (S) prestan especial atención a los detalles y aspectos concretos. Aquellas con preferencia Intuition $(\mathrm{N})$, atienden a los patrones generales y las posibilidades. En cuanto a la toma de decisiones, las personas con preferencia Thinking (T) usan fundamentalmente el análisis lógico e imparcial. En contraste, aquellas con preferencia Feeling $(F)$ toman decisiones basadas en convicciones y valores personales. Los polos de la cuarta dimensión, Judging (J) y Perceiving (P), definen la actitud de las personas frente al mundo. La motivación por concretar las decisiones y vivir de una manera planificada caracteriza a las personas con preferencia J. En contraste, si su preferencia es $\mathrm{P}$, las personas tendrán una disposición más perceptiva y flexible.
Figura 1. Preferencias Psicológicas según el MBTI.

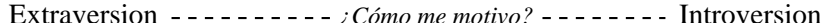

(E)

(I)

Sensing - - - - - - _ ¿Cómo percibo la información? - - - - - Intuition

Thinking - - - - - _ _Cómo tomo decisiones? - - - - - - - - Feeling

Judging - - - - ¿ ¿Cómo me posiciono frente al mundo? - - - Perceiving (J)

(P) 
El cuestionario está constituido por 96 ítemes de elección forzada que representan preferencias en comportamientos cotidianos en cada una de las 4 dimensiones del MBTI. Cada ítem puntúa para uno de los 2 polos de cada dimensión (ej: para E o para I). Para establecer la preferencia de un individuo en una dimensión se comparan los puntos obtenidos en cada uno de los polos. El puntaje mayor indicará la preferencia. Por otra parte, de la combinación de preferencias en cada una de estas 4 dimensiones se origina un patrón de personalidad, denominado tipo psicológico ${ }^{15,23}$, que se representa mediante las 4 letras que individualizan sus preferencias. Por ejemplo, el tipo INTJ caracteriza a una persona cuyas preferencias son Introversion (I), Intuition (N), Thinking (T) y Judging (J). Dieciséis tipos psicológicos surgen de todas las combinaciones posibles. Cada uno de estos tipos tiene fortalezas y debilidades para el aprendizaje, las relaciones con los demás y el desenvolvimiento general en la vida ${ }^{23}$.

Múltiples estudios realizados principalmente en Estados Unidos, indican que el MBTI es un test válido y confiable ${ }^{14,15}$. En general, las 4 dimensiones del test presentan coeficientes de reproducibilidad superiores a 0,8 y los resultados son estables en el tiempo, como lo muestran estudios testretest realizados con intervalos de 3 meses a 2 años ${ }^{14,15}$. Estas propiedades han sido confirmadas en nuestros estudios, como también en un estudio realizado en una muestra representativa de la población urbana de Santiago (Mandiola M, Prat G y Pulido L. "Adaptación y estandarización del MBTI forma $G$ en la población urbana del gran Santiago" (1995) Tesis, Universidad Diego Portales).

Inventario de Estilos de Aprendizaje (IEA). Basado en la Teoría de Aprendizaje Experiencial ${ }^{24}$, el IEA identifica los estilos de aprendizaje según las preferencias de cada individuo por cuatro modos distintos de aprender: la conceptualización abstracta (CA), la experiencia concreta (EC), la observación reflexiva $(\mathrm{OR})$ y la experimentación activa (EA) (Figura 2). CA y EC son modos de adquirir información nueva. Ambos son polos de un continuo, con el pensamiento analítico y formación de conceptos (CA) en un extremo y el aprendizaje experiencial y emocional (EC) en el otro. Por otra parte, en cuanto al procesamiento de la información, la preferencia por escuchar y observar (OR) se ubica en un extremo, y la disposición a actuar o tomar decisiones (EA) en el otro $^{24}$.

La combinación de preferencias para adquirir y procesar la información define cuatro tipos de aprendices (Figura 2). El Asimilador (CA y OR) que tiende a aprender sistematizando la informa-

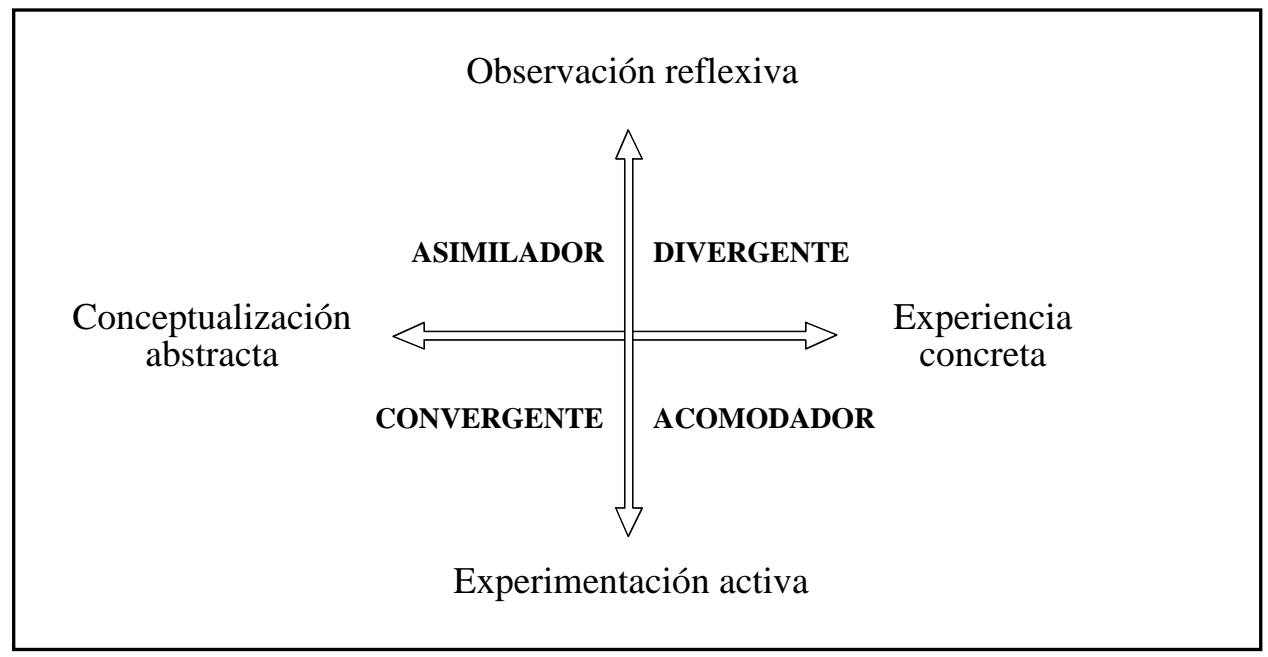

Figura 2. Estilos de aprendizaje según el IEA. 
Tabla 2. Preferencias psicológicas de los estudiantes de medicina

\begin{tabular}{|c|c|c|c|c|c|c|c|c|}
\hline & \multicolumn{8}{|c|}{$\begin{array}{c}\text { Preferencias psicológicas } \\
(\%)\end{array}$} \\
\hline & E & I & $\mathrm{S}$ & $\mathrm{N}$ & $\mathrm{T}$ & F & $\mathrm{J}$ & $\mathrm{P}$ \\
\hline Todos & 52 & 48 & 52 & 48 & 72 & 28 & 73 & 27 \\
\hline Hombres & 50 & 50 & 54 & 46 & 81 & 19 & 69 & 31 \\
\hline Mujeres & 54 & 46 & 50 & 50 & 61 & $39 * * 4$ & 76 & 24 \\
\hline Cohorte 2000 & 51 & 49 & 50 & 50 & 67 & 33 & 69 & 31 \\
\hline Cohorte 2001 & 51 & 49 & $64^{* *}$ & 36 & 69 & 31 & 69 & 31 \\
\hline Cohorte 2002 & 54 & 46 & 42 & 58 & $80^{*}$ & 20 & 80 & 20 \\
\hline
\end{tabular}

${ }^{*} \mathrm{p}=0,05$ comparado con el valor respectivo de la cohorte 2000. ${ }^{* *} \mathrm{p}=0,003$ comparado con el valor respectivo de la cohorte 2002. ${ }^{* *} \mathrm{p}=0,0003$ comparado con el valor respectivo de los hombres.

ción en teorías unificadoras o patrones, y reflexiona acerca de ellos sin mucho interés en su aplicación práctica. El Convergente (CA y EA), que aprende al aplicar el conocimiento a problemas luego de generar modelos hipotéticos. El Divergente (EC y OR), que tiene facilidad para aprender de la experiencia una vez que la ha considerado desde múltiples perspectivas. $\mathrm{Y}$, finalmente, el Acomodador (EC y EA), que aprende mejor haciendo ${ }^{24}$ (Figura 2).

Al clasificar estos 4 aprendices según su modo de adquirir la información, distinguimos dos aprendices abstractos (Asimilador y Convergente) y dos concretos (Divergente y Acomodador). Por otra parte, según su modo de procesar la información, tenemos un par de aprendices reflexivos (Asimilador y Divergente) y otro par activo (Convergente y Acomodador) ${ }^{24}$.

Análisis estadístico. Se utilizaron las pruebas estadísticas t de Student, Chi cuadrado o ANOVA, según correspondiera.

\section{RESULTADOS}

Tipos psicológicos. Analizadas las preferencias psicológicas de los 270 estudiantes (Tabla 2), observamos que igual porcentaje prefiere Extraversion (E) que Introversion (I). Lo mismo ocurre con la percepción de información (S-N). Sin embargo, en la toma de decisiones (T-F) y la postura frente a la vida (J-P), la distribución de los alumnos es desigual. Setenta y dos por ciento utiliza el modo Thinking (T), caracterizado por el análisis lógico, objetivo e imparcial. Por otra parte, $73 \%$ de los alumnos reporta una orientación Judging (J), que define una postura estructurada y decidida frente a la vida (Tabla 2). El par de preferencias TJ caracteriza a $55 \%$ de nuestros estudiantes.

Al estratificar por sexo, observamos que las características de personalidad de hombres y mujeres son similares, excepto en la dimensión TF (Tabla 2). Una mayor proporción de mujeres prefiere el modo Feeling (F) (39\% vs 19\%, $p=0,0003$ ), es decir, privilegia las consideraciones personales y el cuidado por las relaciones humanas. El modo Thinking (T), consecuentemente, es preferido por $81 \%$ de los hombres y $61 \%$ de las mujeres (Tabla 2).

Las 3 cohortes son similares en cuanto a preferencias, aun cuando existen algunas diferencias puntuales (Tabla 2). Específicamente, en la cohorte 2001 hay mayor proporción de estudiantes con preferencia Sensing (S) que en la 2002 $(p=0,003)$. Por otra parte, en la cohorte 2002 hay mayor proporción de estudiantes Thinking $(\mathrm{T})$ que en la cohorte $2000(p=0,05)$.

La distribución de tipos psicológicos de los estudiantes se ilustra en la Figura 3. De los 16 tipos, el más frecuente es el ISTJ (19\% de los estudiantes), seguido por el ESTJ (14\%), el INTJ (11\%) y el ENTJ (11\%). Estos tipos, ubicados en las esquinas de la Figura 3, se caracterizan por su marcada capacidad analítica y resolutiva, por lo que son llamados 'ejecutivos'. El predominio de los 'tipos ejecutivos' 


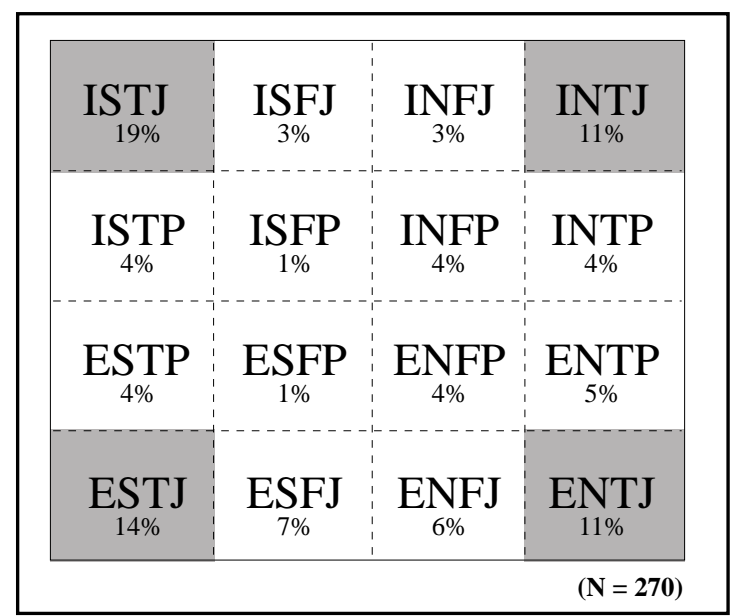

Figura 3. Tipos psicológicos de los estudiantes de medicina

se mantiene ya sea que los datos se analicen separadamente según sexo o cohorte.

Las mujeres tienen mayor proporción de los tipos ESFJs, ISFJs e INFJs, caracterizados por las preferencias F y J ( $p=0,02$; datos no mostrados).
Estilos de aprendizaje. El estilo de aprendizaje más frecuente es el Asimilador, seguido por el Convergente, el Divergente y, finalmente, el Acomodador (Figura 4). Los aprendices abstractos (Asimiladores + Convergentes) concentran 77\% de la población y tienen en común la facilidad para identificar conceptos y relaciones, y para distinguir patrones generales en la información. Los aprendices concretos (Divergentes + Acomodadores) corresponden a $23 \%$ restante y se destacan por la capacidad para usar la experiencia concreta y directa, y para trabajar en equipo.

Desde el punto de vista del uso de la información, el grueso de nuestros estudiantes (68\%) son aprendices reflexivos (Asimiladores + Divergentes), los que se distinguen por examinar acuciosamente las alternativas antes de actuar. Los aprendices activos (Convergentes + Acomodadores), en tanto, corresponden al $32 \%$ restante y tienen en común la tendencia a probar las distintas alternativas sin realizar un análisis previo exhaustivo y a utilizar el conocimiento para la resolución de problemas ${ }^{24}$.

En los hombres hay mayor proporción de aprendices abstractos que en las mujeres ( $81 \%$ vs

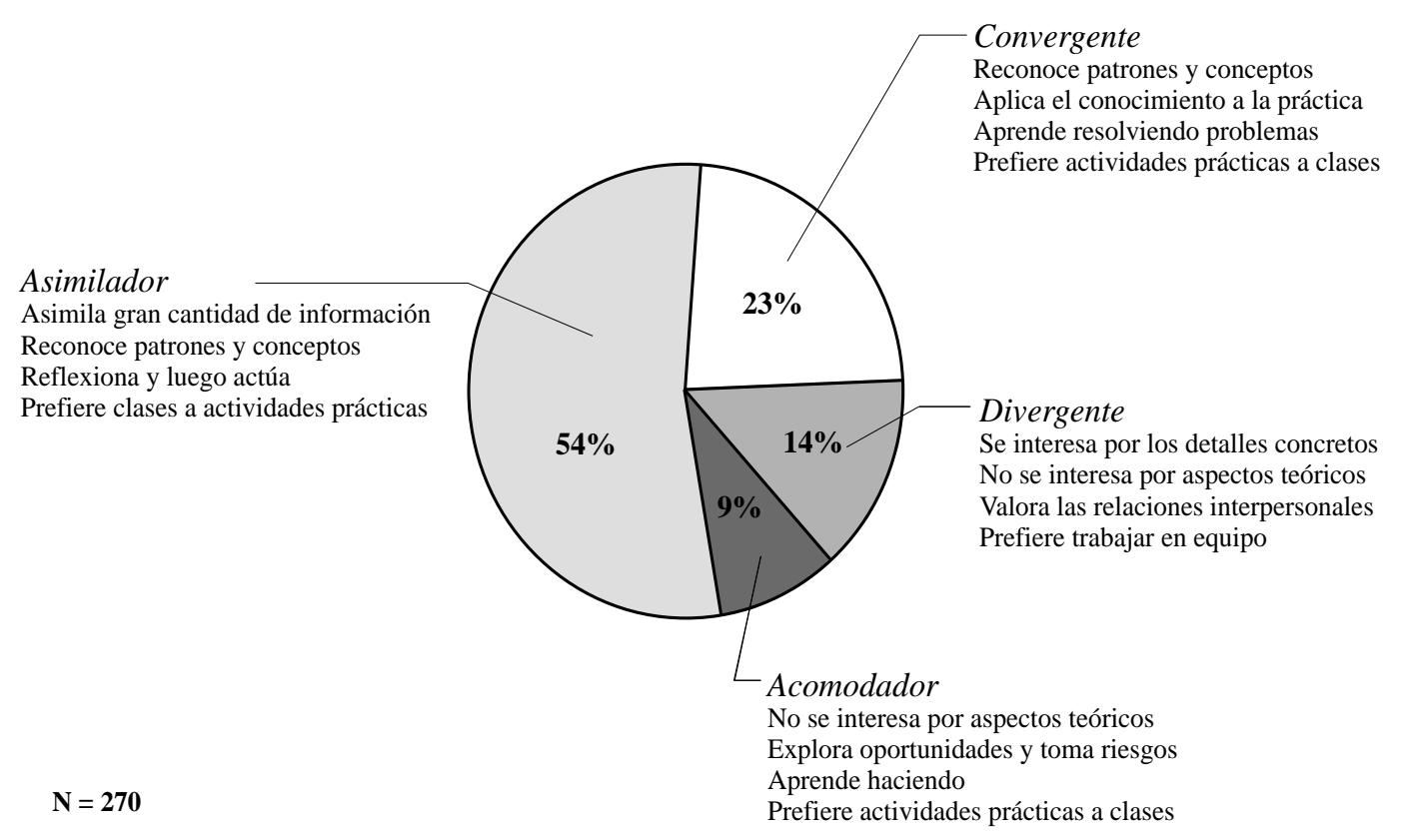

Figura 4. Estilos de aprendizaje de los estudiantes de medicina. 
$72 \% ; p=0,045)$. Por otra parte, en la cohorte 2002 hay mayor porcentaje de aprendices reflexivos que en la 2001 ( $p=0,01$; datos no mostrados).

\section{DisCUSIÓN}

Tipos psicológicos. Los tipos psicológicos predominantes de los estudiantes que ingresaron a medicina en la PUC entre 2000 y 2002 tienen como característica la capacidad de tomar las decisiones basadas en un análisis lógico, objetivo e imparcial de la información ( $\mathrm{T}$ ) y la tendencia a planificar y estructurar su vida, para resolver prontamente los problemas a los que se ven enfrentados (J). Las características menos comunes, por otra parte, son la capacidad para tomar decisiones que privilegien la armonía y el trabajo en equipo (F) y la actitud flexible y abierta ante la vida y sus posibilidades $(\mathrm{P})$.

La extensa investigación de la relación entre tipos psicológicos y educación ${ }^{15}$ indica que los estudiantes con tipos TJ se motivan con actividades académicas bien organizadas y con reflexiones acerca del 'por qué' más que del 'qué'. Estos estudiantes tienen una aproximación sistemática y metódica al estudio. Suelen ser autoexigentes, competitivos e independientes y tienen alta autoestima académica. Valoran en sus profesores el conocimiento 'experto', la imparcialidad y la argumentación lógica ${ }^{15}$

Esta descripción del estudiante TJ coincide con la que nuestros estudiantes hacen de sí mismos. Ellos se consideran, entre otras cosas, "ávidos de saber, "mateos", competitivos, temerosos de la polémica, pasivos e individualistas" 25 . Refieren tener una gran autoexigencia y valoran la nota por sobre lo demás. Esto, declaran, les impide dedicar tiempo a actividades extraacadémicas y redunda en una deficiente calidad de vida ${ }^{25}$.

Nuestro estudio muestra diferencias en la preferencia de mujeres y hombres por la dimensión Thinking - Feeling. Aun cuando en ambos sexos la mayoría prefiere tomar decisiones de un modo Thinking, mayor proporción de mujeres utiliza el modo Feeling. Esto es consistente con múltiples estudios que muestran que la dimensión T-F del MBTI presenta diferencias de género ${ }^{15}$.

A pesar que $72 \%$ de nuestros estudiantes tiene preferencias Thinking, al preguntárseles por el tipo de médico que quieren ser, describen un perfil más bien Feeling. En sus propias palabras, esperan ser médicos que "demuestren preocupación, interés y disposición hacia quien atienden, siendo empáticos y capaces de aceptar a todo tipo de pacientes, guiados siempre por una vocación de servicio"..., "que sean capaces de comunicarse efectivamente y de trabajar en equipo" ${ }^{25}$. Nuestros estudiantes manifiestan la necesidad de desarrollar habilidades interpersonales y de trabajo en equipo, y solicitan a la escuela que fomente modelos de relación docente-alumno más cooperativos y cercanos ${ }^{25}$.

En contraste con los tipos TJ, 13\% de nuestros estudiantes que posee tipos FP (ISFP, ESFP, INFP y ENFP; agrupados en el centro de Figura 3) se motiva con actividades académicas flexibles que tengan espacios de libertad y estén orientadas al servicio y cuidado del prójimo. Tienen una aproximación asistemática y holística al estudio, capacidad para desarrollar proyectos en forma simultánea y cierta dificultad para cumplir plazos. Los estudiantes FP son curiosos e innovadores y prefieren estudiar en grupo. Valoran en sus profesores la capacidad de establecer relaciones personales que les brinden un apoyo sólido ${ }^{15}$.

Existen numerosos estudios de tipos de personalidad en estudiantes de medicina anglosajones, en los que se utiliza el MBTI ${ }^{15,26-28}$. Sin embargo, debido a la disparidad en la forma de presentación de los resultados, no siempre es posible hacer comparaciones estrictas. La mayoría de los estudios reporta únicamente la distribución de las preferencias psicológicas (E-I, S-N, T-F y J-P), mientras que sólo unos pocos indican además la distribución de tipos psicológicos (ej: ISTJ, ISFJ, etc).

Al comparar nuestros estudiantes con estudiantes británicos ${ }^{28}$, encontramos una distribución de preferencias psicológicas idéntica. En ambos casos, más de dos tercios de los estudiantes tiene preferencias Thinking (T) y Judging (J). Esto es también característico de estudiantes de medicina estadounidenses, como muestra un estudio comparativo de 12 escuelas de medicina de USA ${ }^{26}$. Sin embargo, el predominio de las preferencias $\mathrm{T}$ y J es menos marcado en los estudiantes estadounidenses que en los nuestros.

De los 16 tipos psicológicos existentes, el ISTJ es el más frecuente en nuestros estudiantes (19\%). Este resultado es comparable al obtenido en un 
estudio de 1.800 estudiantes de una escuela de medicina norteamericana, entre quienes el tipo ISTJ también resultó ser el más frecuente $(14 \%)^{26}$. Los estudiantes ISTJ son organizados, sistemáticos y responsables. Se destacan por su paciencia, dedicación a los detalles y mentalidad práctica ${ }^{2,23}$. Son muy 'orientados al logro' y suelen obtener las mejores calificaciones ${ }^{15}$. En general, no destinan tiempo a actividades de esparcimiento ${ }^{15}$. En nuestro estudio, el tipo ISTJ es notablemente menos frecuente entre las mujeres (14\% vs $22 \%$ ). En ellas, en cambio, hay una mayor proporción relativa de tipos con preferencias FJ.

No sabemos cómo es el perfil de nuestros estudiantes en comparación con el de los estudiantes chilenos en general, pues no existen comunicaciones en la literatura. Sin embargo, sabemos que nuestros estudiantes se diferencian de los estudiantes que ingresaron a psicología y arquitectura en la PUC en igual período (Zúniga et al., manuscrito en preparación). Por otra parte, dado que los tests se administraron al ingreso, los resultados no son atribuibles al hecho de estudiar medicina. Tampoco resultan del azar, que determinaría una distribución homogénea de 6,25\% de la población en cada tipo psicológico. Desde esta perspectiva, podemos afirmar que en nuestros estudiantes están sobrerrepresentados los tipos con preferencias $\mathrm{T}$ y J y subrepresentados aquellos con preferencias F y P.

Pensamos que estudiar medicina puede ser atractivo para estudiantes $\mathrm{TJ} \mathrm{y}$, particularmente, ISTJ. La idea que ciertos tipos psicológicos se sientan atraídos por determinadas actividades y se autoseleccionen para ellas ha sido planteada tanto a partir de la teoría como de resultados experimentales ${ }^{27}$. Según esta teoría, el atractivo intrínseco de cualquier trabajo o profesión (a diferencia de atractivos extrínsecos como el dinero o el status) reside en la oportunidad que éste brinda para usar las preferencias psicológicas o procesos mentales que dominamos mejor $23,27$.

Estilos de aprendizaje. La mitad de nuestros estudiantes son Asimiladores. Esto significa que se interesan por los conceptos abstractos más que por las aplicaciones prácticas, sopesan exhaustivamente las alternativas antes de actuar y prefieren las disertaciones, clases expositivas y exploración de modelos analíticos ${ }^{24}$. Un cuarto (los Convergentes), también percibe los conceptos y forma teorías, pero se distingue por su interés en el uso práctico de las ideas. En situaciones de aprendizaje formal, prefiere las simulaciones, ensayos de laboratorio y aplicaciones prácticas ${ }^{24}$. El resto (23\%) son aprendices concretos (Divergentes y Acomodadores). Éstos, sin mayor interés por los aspectos teóricos, sobresalen por el cuidado de los detalles, el interés en las relaciones interpersonales y la capacidad para trabajar en equipo ${ }^{24}$.

Entre las mujeres hay mayor proporción de aprendices concretos ( $28 \%$ vs $19 \%$ ). Esta diferencia se ha comunicado previamente, tanto en estudiantes de medicina ${ }^{29}$, como en la población general $^{30}$. La experiencia concreta, propia de los aprendices concretos, al igual que el Feeling se asocia a las mujeres. Las personas que utilizan la experiencia concreta se caracterizan por el compromiso personal y la confianza en los sentimiento $^{24}$. Esta asociación también ocurre en nuestros estudiantes, en el sentido que los aprendices concretos tienen mayor preferencia por Feeling.

En resumen, la mayoría de nuestros estudiantes usa modos abstractos para aprehender la información (77\%) y aborda la toma de decisiones desde una actitud observadora y reflexiva (68\%). En cuanto a sus estilos, nuestros estudiantes son idénticos a los de la Universidad de Concepción (Palacios et al., Segundo Congreso Internacional de Educación en Ciencias de la Salud. ASOFAMECH. Temuco, Chile. Abstracts 2003; p. 59) y muy parecidos a los estudiantes de medicina de la Universidad de Brasilia ${ }^{31}$. Sin embargo, la proporción de aprendices activos (Convergentes y Acomodadores) es menor en nuestros estudiantes que en los estudiantes de medicina norteamericanos ${ }^{32-34}$. Esta diferencia puede deberse a múltiples factores, tales como el tipo de instrucción previa (Enseñanza Media vs College), los criterios de admisión de las distintas escuelas, la edad y características socioculturales de los postulantes, etc.

La inexistencia de estudios normativos de estilos de aprendizaje en estudiantes chilenos no permite hacer comparaciones. Sin embargo, los estudiantes de medicina se diferencian de los de psicología, arquitectura y periodismo (Zúñiga et al, manuscrito en preparación) en que los primeros tienen una menor proporción de aprendices concretos. Estos resultados son consistentes con la 
literatura que indica que, en general, las ciencias básicas atraen preferentemente a aprendices abstractos, mientras que las carreras artísticas y humanistas a aprendices concretos 24,29 .

A pesar de las diferencias descritas, en general existe un predominio de los estilos abstractos entre estudiantes de medicina de distintos países. Esto ha llevado a algunos autores a sugerir que el currículo debiera mantener entre sus metodologías las clases expositivas, que son una forma tradicional de enseñanza particularmente adecuada para estos aprendices ${ }^{29}$. Desde la perspectiva del rendimiento académico, parece razonable esperar que los estudiantes con habilidades abstracto-reflexivas tengan ventajas en el ciclo básico de la carrera y los concreto-activos en el ciclo clínico e internado. De hecho, se han reportado diferencias en la habilidad diagnóstica de aprendices abstractos y concretos ${ }^{31}$, destacándose los abstractos por su capacidad de estructurar el pensamiento y los concretos por su flexibilidad de pensamiento.

\section{CONCLUSIONES GENERALES}

En resumen, de cada 10 estudiantes que ingresan a estudiar medicina en la PUC, 7 se caracterizan por analizar la información de un modo lógico, objetivo e imparcial o por tener una postura estructurada y decidida en la vida. El fuerte de estos estudiantes es la capacidad de asimilar gran cantidad de información y abstraer los conceptos y patrones generales. Tienen una tendencia más reflexiva que activa, por lo que evalúan exhaustivamente las alternativas de acción.

Por otra parte, $30 \%$ que posee el perfil opuesto, basa sus decisiones en consideraciones personales, privilegia la armonía entre las personas, y tiene una actitud flexible y abierta ante la vida y sus posibilidades. Las fortalezas de estos estudiantes residen en su capacidad para aprender de la experiencia directa y trabajar en equipo; en su aprecio y cuidado por los detalles, y en su valoración de las relaciones interpersonales.

Sin lugar a dudas, las caracteństicas de personalidad y estilos de aprender atraviesan la vida académica de cada estudiante, influyendo en la adquisición tanto de conocimientos como de habilidades y actitudes ${ }^{16,35}$. De la adecuación de las características de personalidad y estilos de aprendizaje del estudiante al sistema de enseñanza de la escuela, dependerán sus particulares dificultades y facilidades, éxitos y fracasos. Más aún, el perfil de un estudiante puede ser muy adecuado para enfrentar los desafíos de un ciclo de la carrera pero no para otro. Así, un estudiante 'exitoso' en el ciclo básico de la carera de medicina, puede tener dificultades importantes durante el internado si no cuenta entre sus fortalezas con la facilidad para integrar la información o la motivación y habilidades necesarias para relacionarse con otras personas y trabajar en equipo.

Desde el punto de vista del docente, el conocer la diversidad de estilos de sus alumnos, más que dificultar su labor, puede enriquecerla. Al poner las fortalezas de cada estudiante al servicio del aprendizaje, generará experiencias significativas y favorecerá el trabajo en equipo.

El respeto por las diferencias individuales es particularmente importante en la formación de los médicos. Dada la gran diversidad de ámbitos de conocimiento y acción de la medicina, es preciso contar con médicos de los más variados perfiles personales y profesionales para satisfacer las múltiples y crecientes necesidades de salud de la comunidad.

Junto con ofrecer algunas respuestas, este estudio abre numerosas interrogantes: ¿Son los estudiantes de medicina distintos a la población general? ¿Por qué este tipo de estudiantes elige estudiar medicina? ¿Son estos resultados generalizables a todos los estudiantes de medicina? ¿Son estables las características de personalidad y estilos de aprendizaje? ¿Cómo se relacionan con el perfil profesional del médico que egresa? ¿Tienen alguna relación con la elección de especialidad?

El estudio de las características de personalidad ocupa en la actualidad un lugar importante en la educación médica ${ }^{16,36}$. Más de $30 \%$ de las escuelas de USA considera los tipos psicológicos de sus estudiantes como un antecedente académico importante ${ }^{36}$. De hecho, la American Association of Medical Colleges (AAMC) ha incorporado el uso del Inventario de Tipos Psicológicos de Myers Briggs (MBTI) en su programa MEDcareers, destinado a asesorar a los estudiantes en la elección de especialidad ${ }^{36}$.

En suma, esperamos que este estudio estimule a los educadores a conocer los estilos de sus estudiantes y a hacer un uso constructivo de las diferencias individuales. 


\section{REFERENCIAS}

1. Newble D, ENTwistle N. Learning styles and approaches: implications for medical education. Med Educ 1986; 20: 162-75.

2. LESTER W. Assessing the Psychological Types of Specialists to assist students in career choice. Acad Med 1995; 70: 932-3.

3. McGrath E, Zimet N. Female and male medical students: differences in specialty choice selection and personality. J Med Educ 1977; 52: 293-300.

4. Shen H, Comrey L Predicting medical students' academic performances by their cognitive abilities and personality characteristics. Acad Med 1997; 72: 781-6.

5. Tutron P. Psychometric test results associated with high achievement in basic science components of a medical curriculum. Acad Med 1996; 72: 181-6.

6. Wawck M, Cambre K, Randall H. Personality type and medical specialty. J La State Med Soc 1999; 151: 463-9.

7. Westerman G, Grandy T, Combs C, Turner C. Personality variables as predictors of performance for first year dental students. J Dent Educ 1989; 53: 233-7.

8. Bastías G, Viliarroel L, Zúñiga D, Marshall G, Velasco N, Mena B. Desempeño Académico de los estudiantes de Medicina: ¿Un resultado predecible? Rev Méd Chile 2000; 128: 671-8.

9. García R. Rendimiento Académico en el 1er año y su relación con las variables de ingreso en las Escuelas de Medicina. Rev Méd Chile 1995; 123: 28-36.

10. RoJAs V. Capacidad predictiva del rendimiento académico en ciertas variables detectadas en el alumno, ingreso 1986 de la Escuela de Medicina. Serie Apuntes de Educación Médica 1990; 7: 814.

11. Rosas R. Exito académico universitario: Problemas de su definición, medición y predicción. Psykhe 1992; 1: 25-41.

12. Contreras R, Ubila S, Ugalde H, Vicentini E. Estudio comparativo de la Prueba de Aptitud Académica y las notas de egreso de los estudiantes de la Escuelas de Medicina chilenas. Rev Méd Chile 1984; 112: 1033-43.

13. Bitran M, Wright A, Zúñiga D, Mena B, Velasco N, Moreno R. Mejoría en el desempeño académico de estudiantes de medicina en tiempos de reforma curricular. Rev Méd Chile 2002; 130: 43745.

14. BRIGgs MyerS I. MBTI: Inventario Tipológico Forma G. Manual. Madrid: TEA Ediciones. 1991.

15. Myers I, MacCauley M, Quenk N, Hammer A. MBTI Manual: A guide to the development and use of de Myers-Briggs Type Indicator. Palo Alto, California: Consulting Psychologist Press, Inc. 1998.

16. CuRRY L Cognitive and learning styles in medical education. Acad Med 1999; 74: 409-13.

17. Informe: Estudio comparativo entre la Pontificia Universidad Católica y las demás instituciones de educación superior. Pontificia Universidad Católica de Chile. Vicerrectoría Académica. Dirección General Estudiantil. PUC, Abril 1992.

18. Informe: Alumnos de la Pontificia Universidad Católica de Chile. Pontificia Universidad Católica de Chile. Dirección de Admisión y Registros Académicos (DARA). PUC, Junio 2002.

19. Informe: Alumnos matriculados vía admisión ordinaria y su procedencia escolar. Pontificia Universidad Católica de Chile. Dirección de Admisión y Registros Académicos (DARA). PUC, Abril 2002.

20. Informe: Estudio comparativo entre la Pontificia Universidad Católica de Chile y las demás instituciones de Educación Superior adscritas al Honorable Consejo de Rectores. Pontificia Universidad Católica de Chile. Dirección de Admisión y Registros Académicos (DARA). PUC, Abril 2002.

21. Kоцв D. Inventario de los estilos de aprendizaje: Inventario autoevaluativo y su interpretación. TRG Hay/Mc Ber. 1981.

22. Jung C. Psychological Types. R.F.C. Hull, trans., Collected Works of C.G. Jung, Vol. 6 Bollingen Series XX, Princeton University Press 1977.

23. Myers I, Myers P. Gifts differing: understanding personality type. Palo Alto. California: Davies Black Publishing, 1995.

24. Kоцв D. Experiential Learning: Experience as the Source of Learning and Development. New Jersey: Prentice Hall, PTR. 1984.

25. Benavente A, Salvatierra L, García-Huidobro D. Percepción de los estudiantes de medicina de la Pontificia Universidad Católica de Chile acerca del perfil de médico esperado y su malla curricular actual. Informe del Centro de Estudiantes de Medicina (CEMUC) a la Dirección de la Escuela, Julio 2002. 
26. WaшcK M, Cambre K. Personality Types in Academic Medicine. J La State Med Soc 1999; 151: 378-83.

27. Stilwell N, Wamck M, Thal S, Burleson J. MyersBriggs type and medical specialty choice: a new look at an old question. Teach Learn Med 2000; 12: $14-20$.

28. Clack G, Head J. The personality 'types' of medical students and the implications for medical education. Medical Teacher 1998; 20: 57.

29. Davis N, Hanna L A comparative analysis of the learning style preferences of medical students and practicing physicians using Kolb's learning styles inventory. Humanities and Social Sciences 1999; 59: 4410.

30. KolB D. LSI Learning Style Inventory. Technical Specifications. TRG Hay/Mc Ber. 1995.

31. So BRAL D. Diagnostic ability of medical students in relation to their learning characteristics and preclinical background. Med Educ 1995; 29: 278-82.
32. Engleberg N, Schwen K, Gruppen L. Learning styles and perceptions of the value of various learning modalities before and after a $2^{\text {nd }}$ year course in microbiology and infectious diseases. Teach Learn Med 2001; 13: 253-7.

33. Lynch T, Wowlfl N, Steele, D, Hanssen D. Learning Style Influences Student Examination Performance. The Am J Surg 1998; 176: 62-6.

34. Plovnick M. Primary Care Career Choices and Medical Student Learning Styles. J Med Educ 1975; 50: 849-55.

35. CURRY L Integrating concepts of cognitive or learning style: A review with attention to psychometric standards. Otawa, ON: Canadian College of Health Service Executives. 1987.

36. RICHARD G. The MEDCareers and the role of Type. APT XIV International Conference. Minneapolis, Minnesota. Type and Health Care Symposium, June 28, 2001. 